\title{
Correction to: Out-of-Phase Imaging after Optical Modulation (OPIOM) for Multiplexed Fluorescence Imaging Under Adverse Optical Conditions
}

\section{Raja Chouket, Ruikang Zhang, Agnès Pellissier-Tanon, Annie Lemarchand, Agathe Espagne, Thomas Le Saux, and Ludovic Jullien}

Correction to:

Chapter 13 in: Eli Zamir (ed.), Multiplexed Imaging:

Methods and Protocols, Methods in Molecular Biology, vol. 2350, https://doi.org/10.1007/978-1-0716-1593-5

The chapter was inadvertently published with a typo error in the Table 13.2 for the line "ffDronpa", molar extinction coefficient as "11 $000 \mathrm{M}-1 \mathrm{~cm}-1$ " instead of "105000 M-1cm-1".

The correction has been incorporated by changing the molar extinction coefficient from "11000 M-1cm-1"to "105000 M-1cm-1". 